PUPT-1938

HUTP-00/A023

hep-th/0006085

\title{
1+1 Dimensional NCOS and its $U(N)$ Gauge Theory Dual
}

\author{
Igor R. Klebanov \\ Joseph Henry Laboratories \\ Princeton University \\ Princeton, New Jersey 08544 \\ Juan Maldacena \\ Lyman Laboratories \\ Harvard University \\ Cambridge, MA 02138
}

\begin{abstract}
We study some aspects of open string theories on D-branes with critical electric fields. We show that the massless open string modes that move in the direction of the electric field decouple. In the $1+1$ dimensional case the dual theory is $U(N)$ SYM with electric flux, and the decoupling of massless open strings is dual to the decoupling of the $U(1)$ degrees of freedom. We also show that, if the direction along the electric field is compact, then there are finite energy winding closed string modes. They are dual to Higgs branch excitations of the SYM theory, and their energetics works accordingly. These properties provide new non-trivial evidence for the duality.
\end{abstract}

June 2000 


\section{Introduction}

We study some aspects of non-commutative open string theories. These are open string theories on branes in the presence of a critical electric field. More precisely, they are defined through a scaling limit where the electric field is taken to its critical value [1, 2] (for earlier work, see [3], and for other recent work [4, 5, 6, 7, 8]). The resulting open string theory is the same as the usual open string theory but with a new product which includes Moyal phases in the directions of the electric field, which we take to be the 01 directions. The Moyal phase is determined in terms of the string scale $\alpha_{e}^{\prime}$.

This open string field theory can be defined in various dimensionalities. We concentrate our discussion on the $1+1$ dimensional case. In this case the theory on a D1 brane with $N$ units of electric flux is related to a $U(N)$ SYM theory in a sector with one unit of electric flux [9, 3, 10]. $N$ is related to the open string coupling through the formula $G_{0}^{2}=\frac{1}{N}$. Thus, $G_{0}^{2}$ cannot exceed 1 and at this maximum value the NCOS actually becomes free [11. To achieve weak string coupling, we need to take $N$ to be large. In this way, the $1+1$ dimensional NCOS provides a new connection between large $N$ gauge theory and weakly coupled strings. This duality has very interesting predictions for the open string field theory.

- The gauge theory dual to the NCOS has gauge group $U(N)=U(1) \times S U(N) / Z_{N}$ theory. This implies that we have a free $U(1)$ theory. We stress that it is free at all energies, not just low energies. The $S U(N) / Z_{N}$ theory with one unit of $Z_{N}$ flux has a mass gap [12]. We show in this paper that the massless open strings in $1+1$ dimensional NCOS decouple and give the free $U(1)$ predicted by the duality. All other open string modes are massive: they describe excitations in the $S U(N) / Z_{N}$ theory.

- The field theory has a Higgs branch which has a positive energy density. This implies that if we compactify the field theory on a circle then we should observe a continuum above a certain calculable threshold corresponding to breaking $U(N) \rightarrow U(N-1) \times$ $U(1)$ by a Higgs vev. The low energy excitations should now reflect the presence of massless $U(1)$ modes. We show that the NCOS theory couples to closed strings that wind around the circle with only one orientation. The spectrum of these closed strings is precisely as expected from the duality.

- Let us break $U(N) \rightarrow U(N-K) \times U(K)$ by a Higgs vev, keeping the electric flux in the $U(N-K)$ part. We expect that the low energy dynamics of $U(K)$, for a large Higgs vev, should be given by the Matrix strings [13], i.e. by an orbifold $\operatorname{Sym}\left(R^{8}\right)^{K}$ perturbed by a dimension 3 operator which depends on the Yang Mills coupling but not on $N$ or $K$. We check that the low energy interactions of the closed string modes that couple to NCOS theory have precisely the correct coupling.

- We could consider the gauge theory with $M$ units of electric flux. This corresponds to having $M$ D-branes in NCOS theory. We check that the energetics are as expected. 
After completing these checks of the duality, we make some remarks on the relation of this NCOS theory and the Matrix theory, where the $U(1)$ flux is related to D0 branes. In fact we show that NCOS theory in $1+1$ dimensions is related to DLCQ of IIA string theory in the presence of D0 branes. We also make some remarks on the high-energy and finite temperature behaviors of the NCOS theory.

\section{The duality}

In this section we summarize some aspects of the duality between $1+1$ dimensional NCOS theory and the $U(N)$ SYM [10].

Non commutative open string theory (NCOS) is the theory that results from introducing a Moyal phase $\theta=2 \pi \alpha_{e}^{\prime}$ in the usual open string field theory with parameters $\alpha_{e}^{\prime}, G_{0}^{2}$ [1, 2]. It is useful to view this theory as arising from a decoupling limit of usual string theories. These decoupling limits always involve $g_{s} \rightarrow \infty$ so that to properly analyze them we should go to S-dual descriptions. From this dual description we find that in the $1+1$ dimensional case the decoupling limit reduces to the usual decoupling limit for $N$ D-branes in the presence of a weak electric field [10] which gives a $U(N)$ super Yang Mills theory with one unit of electric flux. Here $N$ is the number of units of the electric flux on the D1 brane in the picture where we define the NCOS limit. The subscipt on $\alpha_{e}^{\prime}$ is introduced to distinguish it from the $\alpha^{\prime}$ of the theory that has been used to define a decoupling limit: $\alpha^{\prime}=\alpha_{e}^{\prime}\left(1-F^{2}\right)$, where $F=F_{01}$ is the electric field on the original D1-brane.

Now let us find the relation between the parameters $\alpha_{e}^{\prime}, G_{0}^{2}$ of the NCOS and the parameters $N, g_{Y M}^{2}$ of the gauge theory. In order to do this it is convenient to go to a parent string theory where the $N$ D-branes live and take then the limit $\alpha^{\prime} \rightarrow 0$. If we analize this limit in the S-dual theory we find the NCOS limit. In [10] this limit was discussed. We note here that we are interested in defining a decoupling limit of the theory of a D1 brane in the presence of $N$ units of $U(1)$ flux.

The relation between $\alpha_{e}^{\prime}$ and the Yang-Mills coupling can be found following the steps in the duality to be (note that our convention for $g_{Y M}^{2}$ differs by a factor of $2 \pi$ from the standard one):

$$
g_{Y M}^{2}=\frac{N^{2}}{\alpha_{e}^{\prime}} .
$$

The square of the open string coupling becomes [3, 10]

$$
G_{0}^{2}=\frac{1}{N} .
$$

More generally we could consider $M$ coincident D-branes in NCOS theory. Now the relation between the electric field on $M$ D1-branes and the number $N$ of flux unit is modified compared to the $M=1$ case [14, 11]. The relation between parameters is found to be

$$
g_{Y M}^{2}=\frac{N^{2}}{M^{2} \alpha_{e}^{\prime}}, \quad G_{0}^{2}=\frac{M}{N} .
$$


where the dual theory is $U(N)$ SYM with $M$ units of flux. It is interesting that now $G_{0}^{2}$ may be an arbitrary fraction. Properties of the theory should depend sensitively on the value of the numerator and denominator: they should vary discontinuously as a function of $G_{0}^{2}$.

In the rest of the paper we check some of the predictions of this duality.

\section{$3 \quad$ Free the U(1)!}

The $U(1)$ modes of the gauge theory are dual to the massless modes in NCOS: the scalars in the vector representation of $S O(8)$ and their superpartners. One of the striking predictions of the duality is that the massless open string modes should decouple. This prediction is even more striking when we combine it with the statement that all the tree level amplitudes in the NCOS theory are the same as in the usual open string theory except for some Moyal phases. Thus, one might conclude that there must be a contradiction in the above statements since the tree level amplitudes involving massless string states are certainly non-vanishing in the usual string theory. What is important to realize, however, is that the statement about the Moyal phases is a statement about ordered correlation functions of vertex operators which correspond to specific Feynman diagrams. The complete amplitudes are given by suming over all orderings and thus the phases introduce the crucial difference.

Consider the simple case of a four point function. The relevant disk diagram decomposes into 6 possible orderings. There are 2 orderings for 3 of the vertex operators which are fixed at $0,1, \infty$ and the fourth can be integrated over $(-\infty, 0),(0,1)$ or $(1,+\infty)$. These three integrals are nonzero individually but their sum turns out to vanish! We perform these calculations explicitly in Appendix A.

In the rest of this section we will present a general argument for why these amplitudes vanish. This is due to the precise value of the Moyal phase in NCOS and it would not be true away from the $F=F_{c r}$ limit. In this limit the correlation function of world sheet fields at the boundary is given by

$$
\left\langle X^{+}(t) X^{-}(0)\right\rangle=4 \alpha_{e}^{\prime}\left(\log |t|+i \frac{\pi}{2} \operatorname{sign}(t)\right)
$$

where $X^{ \pm}=X^{0} \pm X^{1}$. The crucial observation is that we can think of the right hand side of (田), up to an irrelevant constant, as $\log (t)$, where $\log (t)$ is a holomorphic function of $t$ such that when we go from $t>0$ to $t<0$ it produces the phase difference $i \pi$ that we see in (4). There is of course a question of branch cuts and we have to choose them in a definite way. Let us suppose that we are integrating a given massless vertex operator over the boundary of a disk. Since it is massless the on-shell condition implies $p_{+} p_{-}=0$. Let us assume that $p_{-}=0$. Then we choose all branch cuts in the log in (4) so that they are outside the disk. Then we can deform the contour of integration for $t$ from the boundary of the disk to the interior and make it disappear, so that the resulting amplitude is zero. Notice that we did not have to assume that other vertex operators were also massless: the only assumption was that at least one is massless and we integrate that one first. Notice that this implies that 
three point amplitudes are also zero once we add over all orderings since we can take one of the vertex operators and integrate it dividing by the volume of integration, which is finite if we integrate only one. Alternatively we can argue that massless 3-point functions vanish by factorizing the 4-point functions.

For higher loop diagrams it seems that one needs to sum over the insertion of the massless state on all boundaries of the diagram in order to show that the amplitudes vanish. It would be interesting to analyze it more precisely.

Just in case the reader fears that all amplitudes would be zero we compute a four tachyon amplitude in bosonic NCOS theory in Appendix A, and we show that it is non-vanishing. In general, purely massive correlators do not vanish, which agrees with the expectation that $S U(N) / Z_{N}$ gauge theory has non-trivial dynamics. However, as we show in Appendix A, all massive correlators fall off faster at high energies than in the commutative open string. The $2 \rightarrow 2$ scattering cross-section depends on the energy as $E^{-4}$ away from the poles. This is reminiscent of how it works in the gauge theory: $\sigma \sim g_{Y M}^{4} / E^{4}$.

It should be noted that our argument about the decoupling of massless modes may be extended to $p+1$ dimensional NCOS theory with $p>1$. The statement is that the $U(1)$ modes with momentum purely in the 1 direction (i.e. the direction of space-time noncommutativity) decouple from the theory. In the $p=3$ case, analyzed in [2], the NCOS is dual to a space-space non-commutative Yang-Mills theory with $B_{23} \neq 0$. In general, the $U(1)$ modes do not decouple in a non-commutative theory, but if we have a mode that does not depend on $x^{2}$ and $x^{3}$, then it does decouple because the $U(1)$ theory in the remaining directions is commutative. Therefore, our argument translates into non-trivial checks of other dualities in [10] as well.

\section{$4 \quad$ Higgs branch and closed strings}

In this section we analyze the second prediction of the duality: the existence of a Higgs branch with the right energetics. It is convenient for this discussion to compactify the Yang-Mills theory, and also the NCOS theory, on a circle. So we compactify the direction 1 on a circle of radius $R$. The $S U(N)$ with one unit of $Z_{N}$ flux may be Higgsed to an $S U(N-K) \times U(1)^{K}$ with one unit of $Z_{N-K}$ flux in the first factor. The energy cost for this can be calculated from the BPS mass formula for $(p, q)$ strings in the gauge theory limit. The relevant formula says that in a $U(N)$ theory with $M$ units of electric flux we have a ground state energy of the form

$$
E(M, N)=2 \pi R \frac{g_{Y M}^{2}}{4 \pi} \frac{M^{2}}{N} .
$$

Notice that this gives the energy above the $U(N)$ Yang Mills ground state with no flux. The energy of the Higgs branch we considered above, for which $M=1$, is

$$
E_{K}=E(1, N-K)=\frac{R g_{Y M}^{2}}{2} \frac{1}{N-K} \sim \frac{R g_{Y M}^{2}}{2}\left(\frac{1}{N}+\frac{K}{N^{2}}+\frac{K^{2}}{N^{3}}+\cdots\right)
$$


On this Higgs branch we also expect to have a continuum of excitations, corresponding to configurations where the Higgs field has some momentum in the transverse directions. There are also massless excitations propagating along the $U(1)$ s. Let us consider first the case of $K=1$ : just a single $U(1)$. Then we expect to find an energy spectrum for this $U(1)$ which to leading order in $N$ would be

$$
E_{1}=\frac{R}{2 \alpha_{e}^{\prime}}+\frac{1}{R}\left(N_{L}+N_{R}+\alpha_{e}^{\prime} k^{2} / 2\right)
$$

where we have used the relation (11). Notice that the particular dependence of (7) on $N_{R, L}$ is just stating that we have massless free fields. There is also a level matching condition that $N_{L}-N_{R}$ should be an integer. Notice that in (17) we have used only the second term in the expansion 6. The first term represents the mass of the electric flux line itself:

$$
(2 \pi R) \frac{N}{4 \pi \alpha_{e}^{\prime}}=(2 \pi R) \frac{1}{4 \pi \alpha_{e}^{\prime} G_{0}^{2}} .
$$

From the dependence of the tension on $G_{0}^{2}$ we conclude that the flux line is dual to a D-string in the NCOS (this is not surprising because this is precisely the S-duality relation). Note, however, that (8) is a factor of two smaller than the naive expectation for the D1-brane tension in NCOS. The naive expectation is what we get by just stating that the mass of brane is given by the lowest term of the non-commutative analog of the Born-Infeld action 15, 16. On the other hand, if we just stick to the standard string field theory methods and we determine the mass of the brane in the the manner described below then we get the right value. In order to determine the mass of the brane, imagine that we do not take the complete decoupling limit but that we still keep the original $\alpha^{\prime}$ finite. Then one way of computing the mass of the brane would be to consider the string field $\phi$ that parametrizes the position of the brane and to write its kinetic term as $S=\int d t C(\dot{\phi})^{2}$. Then we relate $\phi$ to the physical displacement $r$ of the brane by considering a configuration with two D-branes and relating the mass of the open string computed in string field theory, which is linear in $\phi$, to the mass we expect if we think we have a string stretching between the branes. Finally we write the action as $S=\int d t M \dot{r}^{2} / 2$ and we read off the mass. The only difference in this procedure between the usual case and the case with the Moyal phases is that the mass of the stretched string as a function of $r$ is different. In usual open string theory it is $m=\frac{r}{2 \pi \alpha^{\prime}}$ while in this case it is $m=\frac{r}{2 \pi \alpha_{e f f}^{\prime} \sqrt{1-F^{2}}}$. Computing the mass using this method we get a mass that diverges since it represents the mass of the $(N, 1)$ string in the parent string theory before taking the decoupling limit. If we subtract from this mass the mass of the $N$ D1 branes then we indeed get (8). It would be nice to derive the D-brane mass formula directly in the NCOS theory. We believe that the arguments relating the cubic string field theory action to the Born Infeld action should fail in the NCOS theory. In fact, we saw that the massless states are free, which is incompatible with a Born-Infeld action. 


\subsection{Closed Strings}

The factor of 2 discussed above is not the only puzzling factor in (6). The tension of the Higgs branch excitation calculated in (四) is $1 /\left(4 \pi \alpha_{e}^{\prime}\right)$. Its scaling allows us to identify it with a closed string wound around the compact circle, except for a factor of 2 . Thus, the duality predicts that, even though the masses of open strings are given by the usual formula, $m^{2}=N / \alpha_{e}^{\prime}$ ( $N$ is the integer oscillator level), the compactified NCOS theory contains wound closed strings of tension $1 /\left(4 \pi \alpha_{e}^{\prime}\right)$, instead of the usual closed strings of tension $1 /\left(2 \pi \alpha_{e}^{\prime}\right)$. In the following we confirm this prediction.

The presence of wound closed strings in NCOS is hardly a surprise: once we compactify NCOS then an open string can stretch around the circle and the two ends can join with a finite probability, since the open string coupling constant is finite. Therefore, the NCOS theory can emit wound closed strings into the bulk. Moreover the argument leading to (6) predicts that these strings can only wind with one orientation: the winding number $K$ cannot be negative. We also expect this from the NCOS theory since the energy necessary to stretch the string in one direction is finite while it is infinite in the other direction in the scaling limit of [四, 国].

In order to show directly that these closed strings are produced we sketch the calculation of the non-planar one loop diagram. The compactness of the 1 direction introduces the crucial modification that allows closed string poles, and also explains why the tension is twice smaller than in the commutative case (in [2] it was shown that there are no closed string poles in the non-compact case).

We consider a one-loop diagram, which is a cylinder. We introduce some vertex operators on one boundary and some on the other boundary so that we have some net momentum $p$ flowing along the cylinder from one boundary to the other. As usual, $p^{1}$ is quantized: $p^{1}=n / R$. We are interested in poles arising from the large $s$ region, where $s$ is the proper time along the closed string channel. These poles must be associated with on-shell closed strings. In presence of the NS-NS field $B_{01}$, the on-shell condition for closed strings with $n$ units of momentum and $m$ units of winding can be derived with standard methods (see, for example, [17):

$$
-\alpha^{\prime}\left(p_{0}\right)^{2}-2 p_{0} B_{01} m R+\frac{(m R)^{2}}{\alpha^{\prime}}\left(1-B_{01}^{2}\right)+\alpha^{\prime}(n / R)^{2}+2\left(N_{L}+N_{R}\right)+\alpha_{e}^{\prime} k^{2}=0,
$$

where $k$ is the transverse momentum. These strings do not acquire infinite energy in the scaling limit $\alpha^{\prime}=\alpha_{e}^{\prime}\left(1-B_{01}^{2}\right) \rightarrow 0$ where $\alpha_{e}^{\prime}$ is held fixed. Terms quadratic in $p_{0}$ and $n / R$ vanish, and we obtain a mass formula of non-relativistic type:

$$
p_{0}=\frac{m R}{2 \alpha_{e}^{\prime}}+\frac{\alpha_{e}^{\prime}}{2 m R} k^{2}+\frac{N_{L}+N_{R}}{m R}
$$

together with the standard level-matching condition $N_{L}-N_{R}=m n$. This formula is in precise agreement with the prediction of the duality (7). Especially striking is the fact that that the tension of the wound strings is indeed $1 /\left(4 \pi \alpha_{e}^{\prime}\right)$. It is crucial that the quadratic 
dependence on $p_{0}$ cancels since it implies that $m>0$ for positive energy states, so that strings can wind only in one sense. Notice also the appearance of a continuum associated to the momentum $k$ in the transverse directions.

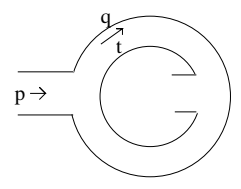

Figure 1: Nonplanar open string diagram. In open string field theory we would build it from the cubic vertex and we would consider states carrying momentum $q$ and $q+p$ along the loop.

We can also see the existence of the closed string poles directly in the open string interpretation of this diagram, in the spirit of the explanation in [2]. The idea is to think about the non-planar diagram in open string field theory where one would have to integrate over the momentum propagating along the loop (see Figure 1). Then the only difference between the NCOS diagram and the usual diagram is the presence of the phase $e^{2 i p \wedge q}$ where $p \wedge q=\pi \alpha_{e}^{\prime}\left(p_{0} q_{1}-p_{1} q_{0}\right), p$ is the momentum flowing through the closed string channel and $q$ is the momentum running along the loop. But now, since momenta are quantized we get that $q_{1}=n / R$. So we integrate over $q_{0}$ and sum over $n$. The rest of the diagram has the same value as in the usual case. For simplicity we set $p_{1}=0$. The propagators can be exponentiated and we have to compute something of the form

$$
\int d q_{0} \sum_{n} e^{i 2 \pi \alpha_{e}^{\prime} p_{0} n / R} e^{-2 \pi \alpha_{e}^{\prime}\left(-q_{0}^{2}+(n / R)^{2}\right) t+\beta p_{0} q_{0}} .
$$

The integral over $q_{0}$ is of the usual form and will produce the usual $\exp \left(+s \frac{\alpha_{e}^{\prime}}{2} p_{0}^{2}\right)$ in the closed string channel (after analytic continuation). The sum over $n$ is the interesting part. By Poisson resummation we will get

$$
\sum_{m} e^{-s \frac{\alpha_{e}^{\prime}}{2}\left|m R / \alpha_{e}^{\prime}-p_{0}\right|^{2}}
$$

We now have to multiply this times the result of the integral over $q_{0}$ times the rest of the string diagram which for small $t$ or large $s$ has the standard expansion in terms of closed strings coming from modular transforming the small $t$ diagram. Putting it all together we have

$$
\sum_{m} \exp \left\{-s\left(\frac{\alpha_{e}^{\prime}}{2}\left(\left|m R / \alpha_{e}^{\prime}-p_{0}\right|^{2}-p_{0}^{2}+k^{2}\right)+2 N\right)\right\} .
$$

We see that the quadratic part in $p_{0}$ cancels and then there is a pole at

$$
p_{0}=\frac{m R}{2 \alpha_{e}^{\prime}}+\frac{\alpha_{e}^{\prime}}{2 m R} k^{2}+\frac{2 N}{m R}
$$


where $N=N_{L}=N_{R}$ which are equal because we assumed $p_{1}=0$. In the case of nonvanishing $p_{1}$ the on-shell condition generalizes to (10).

It is interesting to consider the interaction of these closed strings. From the gauge theory we expect that their low energy interactions are described by a orbifold theory $\operatorname{Sym}\left(R^{8}\right)^{K}$ deformed by a dimension 3 operator with a coefficient of the order of $\frac{1}{g_{Y M}}$ [13]. From the string theory description we expect the same but with a coefficient $G_{0}^{2} \sqrt{\alpha_{e}^{\prime}}$. Fortunately, using (11), (2) we find that the two coincide.

Now let us turn to the interpretation of the third term in (6). The NCOS action will contain a term of the form

$$
S=\frac{R}{\alpha_{e}^{\prime} G_{0}^{2}} \frac{1}{2}\left(2 \pi \alpha_{e}^{\prime}\right)^{2} \dot{A}_{1}^{2} .
$$

For configurations where $A_{1} \sim A_{1}+1 / R$ depends only on time, the Moyal phases do not appear. As in the usual discussion, if we consider a configuration with $K$ units of momentum conjugate to $A_{1}$, then the energy, expanded to quadratic order, will be

$$
E=\frac{R}{\alpha_{e}^{\prime}} \frac{G_{0}^{2} K^{2}}{2} \text {. }
$$

We see from (11), (2) that we reproduce the third term of (61). Since the total winding number for fundamental strings is being kept fixed we find that this $A_{1}$ field will have to carry momentum precisely equal to the number of strings that have been emitted into the bulk.

\subsection{Finite Temperature}

Instead of compactifying the $x^{1}$ direction we could consider the Euclidean theory and compactify $x^{0}$ on a circle of radius $R$. This describes NCOS at temperature $1 /(2 \pi R)$. Considering closed strings wound around the Euclidean time direction, we find the dispersion relation

$$
\alpha^{\prime}\left(p_{1}\right)^{2}+2 i p_{1} B_{01} m R+\frac{(m R)^{2}}{\alpha^{\prime}}\left(1-B_{01}^{2}\right)+\alpha^{\prime}(n / R)^{2}+2\left(N_{L}+N_{R}-1\right)+\alpha_{e}^{\prime} k^{2}=0 .
$$

Notice the tachyonic shift now present in the oscillator energies [18]. In the scaling limit we have

$$
-i p_{1}=\frac{m R}{2 \alpha_{e}^{\prime}}+\frac{\alpha_{e}^{\prime}}{2 m R} k^{2}+\frac{N_{L}+N_{R}-1}{m R} .
$$

As the temperature is increased, a massless closed string state appears at $T=1 /\left(\pi \sqrt{8 \alpha_{e}^{\prime}}\right)$. This is precisely the Hagedorn temperature expected from studying the open string density of states. It would be interesting to study the connection between the closed strings and the NCOS Hagedorn transition in more detail.

\subsection{Coincident D-branes}

Here we consider the case of $M$ coincident D-branes in NCOS theory. The $U(1)$ part of the massless degrees of freedom is free as before. On the other hand we have other nearly 
massless excitations. These excitations might not be truly massless once we include the effects of the interactions. This should be dual to $U(N)$ Yang-Mills theory with $M$ units of flux. In the Yang Mills theory we expect the energy of this configuration to be proportional to

$$
R g_{Y M}^{2} \frac{M^{2}}{2 N} .
$$

Using (3) we find that this agrees with the expected mass of $M$ D-branes in NCOS theory, up to a factor of two mentioned earlier.

A simple observation is that the energy cost to separate them in NCOS theory is zero to leading order in $M / N=G_{0}^{2}$. If $N$ and $M$ are coprime we expect that this marginal direction should be completely lifted. In this case we expect from the field theory that the energy cost to separate them is of order $1 / N^{3} \sim G_{0}^{2} / \alpha_{e}^{\prime}$ (up to factors of order $M$ ). This is an effect that should arise at one loop. Naively the one loop diagram in NCOS is the same as in the usual string theory, so this effect would vanish. What should probably happen is that the mechanism that ensures that $G_{0}^{2}$ is quantized will in this case produce a $F$ flux on the branes that would give rise to the energy difference. It would be interesting to understand the physics quantizing the coupling.

\section{Conclusions}

In this paper we have carried out some consistency checks on the duality conjectures involving non-commutative open string theory (NCOS). We have mainly focused on the $1+1$ dimensional case which is the only one where the non-commutativity is consistent with the Lorentz invariance (although the parity invariance is obviously broken). In this case the NCOS is obtained in the limit of critical electric field along a D-string, and it is believed to be dual to the maximally supersymmetric $1+1$ dimensional $U(N)$ gauge theory with a single flux quantum. Thus, $1+1$ dimensional NCOS is a new example of gauge field/string duality 22, 23.

In the SYM theory the $S U(N) / Z_{N}$ dynamics has a mass gap while the $U(1)$ part decouples at all energy scales. Perhaps our most striking result is that the dual NCOS respects this decoupling through the vanishing of all amplitudes involving massless particles.

We have also considered the NCOS with a compact spatial dimension along the electric field, and showed that in this case open strings couple to winding modes of the closed string sector. The mass formula for such closed strings is in accord with the energetics on the Higgs branch of the gauge theory: the bulk closed strings are dual to individual D-strings which have become liberated from the $(1, N)$ bound state.

After some number of such closed strings have been liberated, their assembly gives rise to a Matrix string theory set-up [13]. Indeed, $1+1$ dimensional maximally supersymmetric SYM theory appeared in the Matrix description of type IIA string theory [13]. It was observed there that one unit of flux in the Yang Mills theory corresponded to a D0 brane of type IIA in the infinite momentum frame. The large $N$ simplifications that we are seeing both in 
the open string theory and closed string theory are the simplifications that happen for the DLCQ limit of string theory. In particular we are analyzing a D0 brane moving at almost the speed of light. Indeed after T-dualizing along 1 direction, critical electric field becomes the velocity equal to the speed of light [14, 1].

It is also interesting to notice an analogy between NCOS and the SL(2,R) WZW model. In the $\mathrm{SL}(2, \mathrm{R}) \mathrm{WZW}$ model, one has short strings and long strings 20]. The short strings are analogous to the open strings of NCOS and the long strings, which can wind only in one direction, are analogous to the closed strings in NCOS. In both cases the coupling constant is quantized, at least in the supersymmetric context. This is not a coincidence since both theories are describing strings in critical electric fields or $H$ fields.

It would be interesting to consider unstable branes in the presence of electric fields. There is an important difference between magnetic field and electric fields on brane-antibrane pair. In the case of magnetic fields the induced $D(p-2)$ charge in the anti-Dp brane is the opposite to that of the $D p$ brane. So the statement that the tachyon condensation is the same in the open string theory with Moyal phases as defined in [19] as in the usual string theory is just a manifestation of Sen's argument [21] that brane antibrane anihillation is universal. In the case that we have a constant electric field on the brane the system has a net fundamental string charge. This implies that after tachyon condensation we should be left with closed strings 21]. It is interesting to notice that we can define the NCOS limit also for unstable branes like Dp-antiDp branes in string theory, or D-branes in bosonic string theory. This will give us an NCOS string theory which has a tachyon. Tachyon condensation in NCOS theory is the same as in the usual string theory since the tachyon condensate is constant. In particular we could consider the case of a D1-anti-D1 pair in the NCOS limit. This theory is dual to a $U(N)$ theory broken to $U(N / 2) \times U(N / 2)$ with opposite $U(1)$ fluxes in each factor. Then we have a relation between parameters as in (3) with $M=2$. If we naively compute the action at the minimum it seems to be off by a factor of two from the expected answer. This factor of two is related to the factor of two present before in the mass of the D-brane. It would be nice to understand this more precisely.

\section{Acknowledgments}

We are grateful to C. Callan, A. Hashimoto, C. Herzog, N. Itzhaki, S. Minwalla, A. Polyakov, A. Strominger, L. Susskind and N. Toumbas for useful discussions. The research of I.R.K. was supported in part by the NSF grant PHY-9802484, and the James S. McDonnell Foundation grant No. 91-48. The research of J.M. was supported in part by DOE grant DEFGO2-91ER40654, NSF grant PHY-9513835, the Sloan Foundation and the David and Lucile Packard Foundation. 


\section{Appendix A}

In this appendix we compute some scattering amplitudes on D1-branes in the presence of electric fields, following [3]. We compute them for a general non-commutativity parameter $\theta$ and then we set $\theta=2 \pi \alpha_{e}^{\prime}$ which corresponds to the NCOS limit.

The simplest nontrivial scattering calculation is the four-point amplitude for massless NS open strings [3]:

$$
\begin{array}{r}
A_{4}\left(\xi_{1}, p_{1} ; \xi_{2}, p_{2} ; \xi_{3}, p_{3} ; \xi_{4}, p_{4}\right) \sim \int\left\{d \sigma_{1} d \sigma_{2} d \sigma_{3} d \sigma_{4}\right\} \\
\left\langle\xi_{1} \cdot V_{0}\left(p_{1}, \sigma_{1}\right) \xi_{2} \cdot V_{0}\left(p_{2}, \sigma_{2}\right) \xi_{3} \cdot V_{-1}\left(p_{3}, \sigma_{3}\right) \xi_{4} \cdot V_{-1}\left(p_{4}, \sigma_{4}\right)\right\rangle
\end{array}
$$

The subindex indicates the picture. The vertex operators for scalar particles (the massless transverse modes of the string) have the form:

$$
\begin{array}{r}
V_{-1}^{j}\left(p_{1}, z\right)=e^{-\phi(z)} \psi^{j}(z) e^{i p_{1} \cdot X(z)} \\
V_{0}^{j}\left(p_{2}, z\right)=\left(\partial X^{j}(z)+i p_{2} \cdot \psi(z) \psi^{j}(z)\right) e^{i p_{2} \cdot X(z)}
\end{array}
$$

where $j=2, \ldots, 9$, while the momenta $p^{\alpha}$ are longitudinal, $\alpha=0,1 . z$ must lie on the real axis.

The Green function on the boundary:

$$
\left\langle X^{\alpha}\left(\sigma_{1}\right) X^{\beta}\left(\sigma_{2}\right)\right\rangle=-2 \alpha_{e}^{\prime} \eta^{\alpha \beta} \ln \left|\sigma_{1}-\sigma_{2}\right|+i \frac{\theta}{2} \varepsilon^{\alpha \beta} \operatorname{sign}\left(\sigma_{1}-\sigma_{2}\right) .
$$

The second term gives rise to phases that depend on the ordering of the operators.

The $1+1$ dimensional massless kinematics is very restrictive. In the center of mass frame the momenta are

$$
p_{1}^{\alpha}=\left(\begin{array}{c}
p \\
p
\end{array}\right), \quad p_{2}^{\alpha}=\left(\begin{array}{c}
p \\
-p
\end{array}\right), \quad p_{3}^{\alpha}=\left(\begin{array}{c}
-p \\
-p
\end{array}\right), \quad p_{4}^{\alpha}=\left(\begin{array}{c}
-p \\
p
\end{array}\right) .
$$

Let us position $V_{2}$ and $V_{4}$ at 0 and $1, V_{3}$ at $\infty$, and integrate over the position of $V_{1}$ from 0 to 1 . This gives the s-channel contribution to the amplitude that was calculated in [3]:

$$
A^{s}=-\frac{G_{0}^{2}}{\alpha_{e}^{\prime}} \delta^{2}\left(\sum_{i} p_{i}\right) \pi s \frac{\cos \left(s \frac{\theta}{2 \alpha_{e}^{\prime}}\right)}{\sin (\pi s)} \xi_{1} \cdot \xi_{3} \xi_{2} \cdot \xi_{4}
$$

with $s$ given by (this corrects a numerical factor in (18) of [3])

$$
s=-t=4 p^{2} \alpha_{e}^{\prime}
$$

So far we have examined the s-channel amplitude, but it turns out that the other channels give comparable contributions. They are not affected by the phases, and their form is

$$
A^{t, u} \sim s^{2} \xi_{1} \cdot \xi_{3} \xi_{2} \cdot \xi_{4} \Gamma(-u)\left[\frac{\Gamma(-s)}{\Gamma(1+t)}+\frac{\Gamma(-t)}{\Gamma(1+s)}\right]
$$


In computing this amplitude we imagine that we artificially add some momentum in the transverse directions as if we were on a higher dimensional brane, so that $u \neq 0$ and then we take the $u \rightarrow 0$ limit. The poles in $u$ cancel and we obtain

$$
A^{t, u}=\frac{G_{0}^{2}}{\alpha_{e}^{\prime}} \delta^{2}\left(\sum_{i} p_{i}\right) \pi s \frac{\cos (\pi s)}{\sin (\pi s)} \xi_{1} \cdot \xi_{3} \xi_{2} \cdot \xi_{4}
$$

Adding this to the s-channel, we have the complete 4-point amplitude

$$
A^{4}=\frac{G_{0}^{2}}{\alpha_{e}^{\prime}} \delta^{2}\left(\sum_{i} p_{i}\right) \pi s \frac{\cos (\pi s)-\cos \left(s \frac{\theta}{2 \alpha_{e}^{\prime}}\right)}{\sin (\pi s)} \xi_{1} \cdot \xi_{3} \xi_{2} \cdot \xi_{4}
$$

For $\theta=0$ this has the correct pole structure, with the $t$ and $u$ channels canceling half of the poles in the s-channel amplitude: they cancel the poles with the residue of the unphysical sign. Remarkably, for $\theta=2 \pi \alpha_{e}^{\prime}$, the amplitude vanishes identically!

We have also calculated some bosonic string 4-point functions involving the massless scalar particles. The amplitude for 4 massless scalars turns out to have exactly the same form as (26). For the forward scattering of a scalar off a tachyon, we find that the complete amplitude is

$$
\frac{G_{0}^{2}}{\alpha_{e}^{\prime}} \delta^{2}\left(\sum_{i} p_{i}\right) \pi(s+1) \frac{\cos [\pi(s+1)]-\cos \left[(s+1) \frac{\theta}{2 \alpha_{e}^{\prime}}\right]}{\sin [\pi(s+1)]} \xi_{1} \cdot \xi_{2},
$$

which again vanishes in the NCOS limit, $\theta=2 \pi \alpha_{e}^{\prime}$.

If all operators are massive, then the amplitude no longer vanishes in general. To check this we have calculated the complete 4-tachyon amplitude on the bosonic D1-brane:

$$
A=-\frac{G_{0}^{2}}{\alpha_{e}^{\prime}} \delta^{2}\left(\sum_{i} p_{i}\right) \pi(s+2) \frac{\cos \left(\frac{\theta}{2 \alpha_{e}^{\prime}} \sqrt{s(s+4)}\right)-\cos (\pi s)}{\sin (\pi s)}
$$

Obviously, this no longer vanishes for $\theta=2 \pi \alpha_{e}^{\prime}$. However, in the NCOS limit the amplitude has much softer $U V$ behavior than the $\theta=0$ amplitude. Indeed, for large $s$ we may approximate

$$
\cos (\pi \sqrt{s(s+4)}) \approx \cos [\pi(s+2)-2 \pi /(s+2)] .
$$

Therefore, for large $s$,

$$
A \sim \frac{G_{0}^{2}}{\alpha_{e}^{\prime}} \delta^{2}\left(\sum_{i} p_{i}\right) \frac{\sin [\pi s-\pi /(s+2)]}{\sin [\pi s]},
$$

which implies that the residues of the poles at $s=n$ fall off as $1 /(n+2)$ for large $n$. Away from the poles, however, the amplitude is energy independent at high energies. This should be contrasted with the commutative open string where this amplitude grows as $E^{2}$.

This softened UV behavior of NCOS massive amplitudes is general. If we consider 4 particles of mass $m$, then the amplitude has a factor

$$
\cos \left(\pi \sqrt{s\left(s-4 m^{2} \alpha_{e}^{\prime}\right)}\right)-\cos (\pi s)
$$


which leads to a cancellation for large $s$.

It is interesting that this behavior of 4-point amplitudes is similar to the energy dependence in the perturbative Yang-Mills theory in $1+1$ dimensions. Away from the poles

the NCOS amplitude is of order $\frac{G_{0}^{2}}{\alpha_{e}^{\prime}}$ in the high-energy limit, so that the $2 \rightarrow 2$ scattering cross-section is

$$
\sigma \sim \frac{G_{0}^{4}}{\left(\alpha_{e}^{\prime} E^{2}\right)^{2}}
$$

The perturbative amplitude for $4 \mathrm{~W}$-bosons is energy-independent and of order $g_{Y M}^{2}$, so that the scattering cross-section is $\sim\left(g_{Y M} / E\right)^{4}$.

\section{References}

[1] N. Seiberg, L. Susskind and N. Toumbas, "Strings in background electric field, space/time non-commutativity and a new non-critical string theory," hep-th/0005040.

[2] R. Gopakumar, J. Maldacena, S. Minwalla and A. Strominger, "S-duality and noncommutative gauge theory, hep-th/0005048.

[3] S. Gukov, I.R. Klebanov and A.M. Polyakov, Phys. Lett. B423 (1998) 64, hepth/9711112.

[4] N. Seiberg, L. Susskind and N. Toumbas, "Space/time noncommutativity and causality," hep-th/0005015.

[5] O. Ganor, G. Rajesh and S. Sethi, "Duality and noncommutative gauge theory," hepth/0005046.

[6] J. Barbon and E. Rabinovici, "Stringy fuzziness as the custodian of time-space noncommutativity," hep-th/0005073.

[7] J. Gomis and T. Mehen, "Space-time noncommutative field theories and unitarity," hep-th/0005129.

[8] T. Harmark, "Supergravity and space-time non-commutative open string theory," hepth/0006023.

[9] H. Verlinde, "Matrix String Interpretation of the Large N Loop Equation," hepth/9705029.

[10] R. Gopakumar, S. Minwalla, N. Seiberg and A. Strominger, "(OM) Theory in Diverse Dimensions," hep-th/0006062.

[11] I.R. Klebanov, Talk delivered at Lennyfest, May 2000. 
[12] E. Witten, "Bound States Of Strings And p-Branes," Nucl. Phys. B460, 335 (1996), hep-th/9510135.

[13] R. Dijkgraaf, E. Verlinde and H. Verlinde, "Matrix String Theory," Nucl. Phys. B500 (1997) 43.

[14] C.G. Callan, I.R. Klebanov, "D-Brane Boundary State Dynamics," Nucl. Phys. B465 (1996) 473-486, hep-th/9511173.

[15] E.S. Fradkin and A.A. Tseytlin, "Nonlinear Electrodynamics From Quantized Strings," Phys. Lett. B163 (1985) 123.

[16] A. Abouelsaoud, C. Callan, C. Nappi and S. Yost, "Open Strings In Background Gauge Fields," Nucl. Phys. B280 (1987) 135.

[17] J. Polchinski, String Theory, vol. 1, sec. 8.4.

[18] J.J. Atick and E. Witten, "The Hagedorn transition and the number of degrees of freedom of string theory," Nucl. Phys. B310 (1988) 291; I. Kogan, "Vortices on the world sheet and string's critical dynamics," JETP Lett. 45 (1987) 709; B. Sathiapalan, "Vortices on the string world sheet and constraints on toral compactification," Phys. Rev. D35 (1987) 3277.

[19] N. Seiberg and E. Witten, "String theory and noncommutative geometry," hepth/9912072.

[20] J. Maldacena and H. Ooguri, "Strings in $\operatorname{AdS}(3)$ and SL(2,R) WZW model. I," hepth/0001053.

[21] A. Sen, "Universality of the tachyon potential," JHEP 9912, 027 (1999) hepth/9911116.

[22] G. 't Hooft, "A planar diagram theory for strong interactions," Nucl. Phys. B72 (1974) 461.

[23] A.M. Polyakov, "String Theory and Quark Confinement," hep-th/9711002. 